\title{
Autopercepção de saúde de idosos que vivem em estado de corresidência
}

\author{
Self concept of health of elderly living with relatives
}

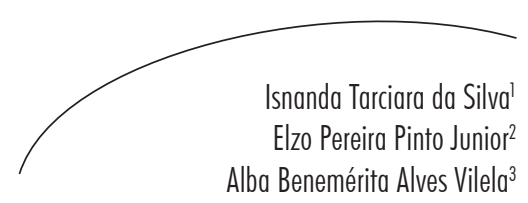

\section{Resumo}

Objetivos: Descrever a autopercepção de saúde e os fatores associados em idosos corresidentes e identificar suas características socioeconômicas e de saúde. Metodologia: Trata-se de um estudo transversal, descritivo, com abordagem quantitativa, realizado com 191 idosos corresidentes, com idade igual ou superior a 60 anos, assistidos pela Estratégia de Saúde da Família em um município do estado da Bahia. Foi aplicado um instrumento de coleta de dados contendo questões sociodemográficas, avaliação funcional, autorrelato de doenças crônicas e autopercepção de saúde. As análises foram feitas com base nas estatísticas descritiva e inferencial, com realização do teste qui-quadrado. Resultados: Constatou-se o predomínio de mulheres $(62,8 \%)$, com mais de 70 anos $(61,3 \%)$, casada $(50,8 \%)$ e com filhos $(92,7 \%)$. Os idosos mostraramse satisfeitos com o arranjo familiar em que viviam $(96,6 \%)$, com a vida que tinham $(85,8 \%)$ e acreditavam ser a corresidência vantajosa para eles e para a família $(79,4 \%)$. Apresentaram autopercepção negativa de saúde $(59,1 \%)$ e independência na realização de atividades da vida diária, tendo sido a hipertensão arterial (70,7\%) a mais prevalente. Como fatores associados à autopercepção de saúde foram encontrados: "nível de ajuda para AIVD”, "presença de doença crônica", "hipertensão arterial", "doença cardíaca", "reumatismo", "doença de coluna" e "diabetes". Conclusão: Observou-se que a maioria da população estudada apresenta autopercepção negativa de saúde, associada à existência de doenças crônicas. Apesar de ser uma avaliação subjetiva, a autopercepção de saúde é um importante instrumento para que se conheçam a situação global e o impacto que gera no bem-estar físico, social e mental.

\footnotetext{
Programa de Pós-graduação em Enfermagem e Saúde. Universidade Estadual do Sudoeste da Bahia. Jequié, BA, Brasil.

2 Programa de Pós-graduação em Saúde Coletiva. Universidade Estadual do Ceará. Fortaleza, CE, Brasil.

Departamento de Saúde. Universidade Estadual do Sudoeste da Bahia. Jequié, BA, Brasil.
}

Apoio financeiro: Universidade Estadual do Sudoeste da Bahia, Fundação de Amparo à Pesquisa do Estado da Bahia (FAPESB) (Processo n 1489/2010) e do Conselho Nacional de Desenvolvimento Científico e Tecnológico (CNPq) por meio da concessão de bolsas de iniciação científica.

Palavras-chave:

Envelhecimento.

Autoimagem. Doença

Crônica.

E-mail: isnanda.fisio@yahoo.com.br 


\section{Abstract}

Objectives: Describe self-rated health and associated factors in elderly living with relatives and identify their socioeconomic and health characteristics. Methodology: This is a descriptive study, with a quantitative approach performed with 191 elderly aged over 60 years, assisted by the Family Health Strategy in a municipality of the state of Bahia, Brazil. A questionnaire was applied to collect data containing questions about social and demographic aspects, functional health evaluation, self-reported chronic conditions and self-rated health. The analyses were based on descriptive and inferential statistics, using the Chi-square test. Results: It was observed a predominance of women $(62.8 \%)$, with more than 70 years (61.3\%), married (50.8\%) and children (92.7\%). They were satisfied with the family arrangement in which they live $(96.6 \%)$, with the life they have $(85.8 \%)$ and believe living with relatives is advantageous for them and the family $(79.4 \%)$. Showed negative self-perception of health (59.1\%) and independence in performing basic activities of daily living (94.2\%). No need assistance for activities of daily living and have at least one chronic disease (87.4\%), and hypertension $(70.7 \%)$ was the most prevalent disease. Factors associated with self-rated health were: " level of help for IADL", "chronic diseases", "hypertension", "heart disease" "rheumatism", "disease" column and "diabetes." Conclusion: It was observed that the majority of the population has a negative selfperception of health, the existence of associated chronic diseases. Although subjective, self-rated health of an individual is an important tool for knowing the global situation and the impact this generates the physical, social and mental well-being.
Key words: Aging. SelfConcept. Chronic Disease.

\section{INTRODUÇÃo}

O envelhecimento populacional é um processo relativamente novo nos países em desenvolvimento socioeconômico, como o Brasil. Nesse contexto, as políticas públicas e os serviços de saúde não se prepararam para esta situação e buscam alternativas políticas e sociais para se adaptar. Uma dessas alternativas é a institucionalização de idosos, que leva à lotação de instituições de longa permanência para idosos e filas de espera para internamento, fatos considerados consequência do não oferecimento de outros modelos de assistência aos idosos pela sociedade. ${ }^{1}$

Outra alternativa utilizada para o acolhimento desse segmento populacional está na corresidência entre familiares e idosos. Assim, a família tem especial relevância por ser o contexto social mais próximo do idoso, e também porque os relacionamentos mantidos por corresidentes em seus arranjos domiciliares têm implicações positivas para a saúde, fornecendo ao idoso apoio formal e informal. ${ }^{2}$

Mesmo que alguns fenômenos suscitem questionamentos sobre o futuro da família na sociedade, suas responsabilidades e funções sociais parecem não ter perdido a relevância, pois a família permanece como elemento-chave para sobrevivência dos indivíduos, transmissão cultural, relações de gênero, afetividade e solidariedade entre gerações. ${ }^{3-5}$

No Brasil, estudos mostram que a ocorrência de corresidência está muito relacionada com as dificuldades econômicas, em que pais idosos e filhos adultos buscam se ajudar mutuamente, fortalecendo assim os laços de obrigação, lealdade e segurança. ${ }^{3}$

Os idosos estão vivendo cada vez mais e nesse processo de longevidade acabam acumulando comorbidades, com impacto direto em suas condições funcionais. Dessa forma, classificálos de acordo com a doença que os acomete não é mais suficiente para descrevê-los de modo integral. Nesse contexto, faz-se necessário estudar a autopercepção de saúde desses idosos, tendo em vista que esta abordagem engloba vários aspectos da saúde física, cognitiva e de capacidade funcional, ao mesmo tempo em que são por eles determinados. ${ }^{6}$ 
A percepção do indivíduo sobre seu próprio estado de saúde tem se transformado em importante marcador do seu bem-estar e qualidade de vida, sendo útil para avaliar as necessidades de saúde e predizer sua sobrevida. A maneira como o indivíduo lida com seu estado de saúde determinará seu comportamento, suas escolhas e seu modo de viver. ${ }^{7}$ Vale acrescentar que a autoavaliação do estado de saúde é considerada confiável e válida frente a outras medidas mais complexas da condição de saúde, apesar do seu caráter subjetivo, mostrandose consistente em estudos populacionais com diferentes características culturais. ${ }^{8,9}$

Este estudo se justifica pela importância da autoavaliação como ferramenta de investigação ampliada do estado de saúde do idoso. A autopercepção de saúde é uma abordagem que permite entender de forma ampliada os aspectos da saúde física e cognitiva do idoso. Seu uso em populações de idosos corresidentes ajuda a compreender o processo saúde-doença sob a ótica do próprio indivíduo e os possíveis impactos que o fato de morar com a família trazem a sua autoimagem.

A relevância deste estudo está em apontar a possibilidade de investigação da condição de saúde do idoso, de forma que sua autopercepção seja considerada pelos profissionais e gestores da saúde. Sendo assim, a pesquisa objetivou descrever a autopercepção de saúde e os fatores associados em idosos corresidentes, bem como identificar suas características socioeconômicas e de saúde.

\section{MÉTODOS}

Trata-se de um estudo transversal, descritivo, com abordagem quantitativa, que foi realizado por meio de inquérito domiciliar com idosos que residem na área de abrangência de uma Unidade de Saúde da Família (USF) num município do interior do estado da Bahia, Brasil. A população estudada vive numa localidade situada na periferia do município, com acesso a diversos equipamentos sociais, como igrejas, quadras poliesportivas, escolas, mercados e pequenas lojas. Conta ainda com saneamento básico na maioria dos domicílios, além de energia elétrica e acesso a linhas urbanas de transporte público.

A pesquisa foi realizada no período de março a junho de 2011 e teve como público-alvo todos os idosos em estado de corresidência, com idade igual ou acima de 60 anos que residiam na área de abrangência dessa USF. Foram excluídos da amostra aqueles que não estavam no domicílio por quatro vezes seguidas em dias e horários alternados, ou que se recusaram a participar da pesquisa e discordaram do Termo de Consentimento Livre e Esclarecido (TCLE).

Na área adstrita à USF foram identificados 293 idosos, dos quais 218 foram entrevistados; destes, 191 (91,8\%) vivem em estado de corresidência e participaram do estudo.

$\mathrm{O}$ inquérito domiciliar foi aplicado por acadêmicos voluntários devidamente treinados para a coleta. Nos casos em que o idoso não tivesse condições de responder, a pesquisa poderia ser respondida pelo familiar, exceto as perguntas acerca da opinião do idoso, como "satisfação com a vida", "satisfação em relação ao arranjo famíliar", "opinião acerca da corresidência" e "autopercepção de saúde".

Esta pesquisa foi precedida por um estudopiloto, realizado com idosos residentes em outra USF do mesmo município, que teve como objetivo testar os instrumentos da coleta de dados. Após o estudo-piloto, foram feitas as adaptações necessárias nos instrumentos, a fim de torná-los mais específicos aos objetivos do projeto.

A versão final do instrumento de coleta de dados consistiu da compilação de cinco questionários de pesquisa em saúde já validados em território nacional. Dentre eles, encontra-se o Brazilian Old Age Schedule (BOAS), desenvolvido para uso em inquéritos epidemiológicos para avaliar aspectos da população idosa, o Índice de Katz e a Escala de Lawton. O instrumento constou ainda de questões sobre prevalência de doenças crônicas, segundo a Pesquisa Nacional por Amostra de Domicílios (PNAD 2003), ${ }^{10}$ que tem por objetivo analisar as doenças 
autorreferidas. Por fim, foi utilizado o estudo Saúde, Bem-Estar e Envelhecimento (SABE),${ }^{11}$ com perguntas sobre autopercepção de saúde, internações hospitalares e quedas.

Os dados foram tabulados no software Epidata v.3.2 e analisados no Statistical Packeage for the Social Sciences, v.15.0. As análises foram feitas com base na estatística descritiva (construção de tabelas de frequência) e na estatística inferencial (teste de qui-quadrado de Pearson). Optou-se por não utilizar medidas de tendência central e de dispersão em algumas variáveis contínuas, a fim de possibilitar sua categorização em variáveis nominais, facilitando a realização de testes estatísticos. Admitiu-se significância estatística entre as variáveis cujo p-valor do teste do quiquadrado fosse menor que 0,05 .
Este estudo foi aprovado pelo Comitê de Ética em Pesquisa da Universidade Estadual do Sudoeste da Bahia, sob o no 047/2009, e seus autores agiram em todas as etapas da pesquisa de acordo com a Resolução no 196/96 do Conselho Nacional de Saúde. Todos os participantes deste estudo assinaram o TCLE.

\section{RESULTADOS}

A tabela 1 apresenta os dados referentes às características sociodemográficas dos entrevistados. Conforme observado, foram encontrados $62,8 \%$ de idosos do sexo feminino, com mais de 70 anos de idade (61,3\%), casados $(50,8 \%)$, com filhos $(92,7 \%)$ e recebendo algum tipo de benefício previdenciário $(80,6 \%)$.

Tabela 1. Dados sociodemográficos de idosos que vivem em estado de corresidência. Jequié-BA, 2011.

\begin{tabular}{lcc}
\hline \multicolumn{1}{c}{ Dados sociodemográficos } & $\mathrm{n}$ & $\%$ \\
\hline Sexo & 71 & 37,2 \\
Masculino & 120 & 62,8 \\
Feminino & & \\
Faixa etária & 74 & 38,7 \\
60-69 anos & 71 & 37,2 \\
$70-79$ anos & 46 & 24,1 \\
80 anos ou mais & & \\
Estado conjugal & 11 & 5,8 \\
Solteiro(a) & 97 & 50,8 \\
Casado(a) & 69 & 36,1 \\
Viúvo(a) & 14 & 7,3 \\
Divorciado(a) & & 92,7 \\
Filhos & 177 & 7,3 \\
Sim & 14 & 75,9 \\
Não & & 19,4 \\
Benefício previdenciário & 9 & 4,7 \\
Aposentadoria e pensão & 145 & \\
Aposentadoria ou pensão & 37 & \\
Nenhum dos dois & & \\
\hline
\end{tabular}


No que se refere aos lares desses idosos, observou-se a maior parte das residências com três ou mais moradores (63,9\%). Grande maioria desses idosos alegou estar satisfeita com o arranjo familiar em que vivem $(96,6 \%)$ e com a vida que têm $(85,8 \%)$, além de acreditarem ser a corresidência vantajosa para eles e para a família $(79,4 \%)$, segundo a tabela 2 .

Tabela 2. Caracterização dos lares nos quais residem idosos em estado de corresidência. Jequié-BA, 2011.

\begin{tabular}{lcc}
\hline \multicolumn{1}{c}{ Caracterização dos lares } & $\mathrm{n}$ & $\%$ \\
\hline Número de moradores & 69 & 36,1 \\
2 moradores & 54 & 28,3 \\
3 moradores & 68 & 35,6 \\
$\quad 4$ ou mais moradores & 172 & \\
Satisfação em relação ao arranjo familiar & 6 & 36,6 \\
$\quad$ Satisfeito & & 3,4 \\
Insatisfeito & 139 & 79,4 \\
Opinião acerca da corresidência & 21 & 12,0 \\
Vantajoso para ambos & 15 & 8,6 \\
Vantajoso para o idoso & & 85,8 \\
Vantajoso para a família & 151 & 14,2 \\
Satisfação com a vida & 25 & \\
Satisfeito &
\end{tabular}

Quanto às condições de saúde, a tabela 3 evidencia que a população estudada apresentou uma autopercepção negativa de saúde (59,1\%); $94,2 \%$ eram independentes para realizar atividades básicas da vida diária (ABVDs); 51,3\% não necessitavam de auxílio para as atividades instrumentais da vida diária (AIVDs); e87,4\% tinham, pelo menos, uma doença crônica. As doenças mais encontradas nesta população foram hipertensão arterial $(70,7 \%)$, doença de coluna $(50,8 \%)$, reumatismo $(50,3 \%)$, doença cardíaca $(19,4 \%)$ e diabetes $(18,3 \%)$. 
Tabela 3. Condições de saúde dos idosos corresidentes. Jequié-BA, 2011.

\begin{tabular}{|c|c|c|}
\hline Condições de saúde & $\mathrm{n}$ & $\%$ \\
\hline \multicolumn{3}{|l|}{ Autopercepção de saúde } \\
\hline Positiva & 74 & 40,9 \\
\hline Negativa & 107 & 59,1 \\
\hline \multicolumn{3}{|l|}{ Avaliação funcional para ABVD } \\
\hline Independente & 180 & 94,2 \\
\hline Dependente & 11 & 5,8 \\
\hline \multicolumn{3}{|l|}{ Nível de ajuda para AIVD } \\
\hline Sem auxílio & 98 & 51,3 \\
\hline Algum tipo de auxílio & 93 & 48,7 \\
\hline \multicolumn{3}{|l|}{ Presença de doença crônica } \\
\hline $\operatorname{Sim}$ & 167 & 87,4 \\
\hline Não & 24 & 12,6 \\
\hline \multicolumn{3}{|l|}{ Hipertensão arterial } \\
\hline $\operatorname{Sim}$ & 135 & 70,7 \\
\hline Não & 56 & 29,3 \\
\hline \multicolumn{3}{|l|}{ Doença cardíaca } \\
\hline $\operatorname{Sim}$ & 37 & 19,4 \\
\hline Não & 154 & 80,6 \\
\hline \multicolumn{3}{|l|}{ Reumatismo } \\
\hline $\operatorname{Sim}$ & 95 & 49,7 \\
\hline Não & 96 & 50,3 \\
\hline \multicolumn{3}{|l|}{ Doença de coluna } \\
\hline $\operatorname{Sim}$ & 97 & 50,8 \\
\hline Não & 94 & 49,2 \\
\hline \multicolumn{3}{|l|}{ Diabetes } \\
\hline $\operatorname{Sim}$ & 35 & 18,3 \\
\hline Não & 156 & 81,7 \\
\hline
\end{tabular}

$\mathrm{Na}$ análise com base na estatística inferencial dos fatores associados à autopercepção de saúde, encontrou-se associação estatisticamente significante com as variáveis "nível de ajuda para AIVD" (p-valor=0,006); "presença de doença crônica" ( $p$-valor $<0,001)$; "hipertensão arterial" (p-valor $<0,001)$; “doença cardíaca" ( $\mathrm{p}$-valor=0,005); "reumatismo" ( $\mathrm{p}$-valor $<0,001)$; "doença de coluna" ( $\mathrm{p}$-valor $<0,001)$ e "diabetes" $(\mathrm{p}$-valor $=0,001)$, estando esses fatores associados a uma pior autoavaliação de saúde, conforme mostra a tabela 4. 
Tabela 4. Relação entre autopercepção do estado de saúde dos idosos corresidentes e características sociodemográficas, aspectos sociais, nível funcional e doenças crônicas. Jequié-BA, 2011.

\begin{tabular}{|c|c|c|c|c|c|}
\hline \multirow{3}{*}{ Características dos idosos } & \multicolumn{4}{|c|}{ Autopercepção de saúde } & \multirow{3}{*}{ p-valor } \\
\hline & \multicolumn{2}{|c|}{ Positiva } & \multicolumn{2}{|c|}{ Negativa } & \\
\hline & $\mathrm{n}$ & $\%$ & $\mathrm{n}$ & $\%$ & \\
\hline Sexo & & & & & 0,849 \\
\hline Masculino & 28 & 41,8 & 39 & 58,2 & \\
\hline Feminino & 46 & 40,4 & 68 & 59,6 & \\
\hline Faixa etária & & & & & 0,956 \\
\hline $60-69$ anos & 30 & 42,3 & 41 & 57,7 & \\
\hline 70-79 anos & 28 & 40,0 & 42 & 60,0 & \\
\hline 80 anos ou mais & 16 & 40,0 & 24 & 60,0 & \\
\hline Satisfação em relação ao arranjo familiar & & & & & 0,694 \\
\hline Satisfeito & 71 & 41,3 & 101 & 58,7 & \\
\hline Insatisfeito & 3 & 50,0 & 3 & 50,0 & \\
\hline Satisfação com a vida & & & & & 0,299 \\
\hline Satisfeito & 65 & 43,0 & 86 & 57,0 & \\
\hline Insatisfeito & 8 & 32,0 & 17 & 68,0 & \\
\hline Nível de ajuda para AIVD & & & & & $0,006^{*}$ \\
\hline Sem auxílio & 48 & 50,5 & 47 & 49,5 & \\
\hline Algum tipo de auxílio & 26 & 30,2 & 60 & 69,8 & \\
\hline Presença de doença crônica & & & & & $<0,001^{*}$ \\
\hline $\operatorname{Sim}$ & 56 & 35,4 & 102 & 64,6 & \\
\hline Não & 18 & 78,3 & 5 & 21,7 & \\
\hline Hipertensão arterial & & & & & $<0,001^{*}$ \\
\hline Sim & 40 & 31,7 & 86 & 68,3 & \\
\hline Não & 34 & 61,8 & 21 & 38,2 & \\
\hline Doença cardíaca & & & & & $0,005^{*}$ \\
\hline $\operatorname{Sim}$ & 7 & 20,0 & 28 & 80,0 & \\
\hline Não & 67 & 45,9 & 79 & 54,1 & \\
\hline Reumatismo & & & & & $<0,001^{*}$ \\
\hline $\operatorname{Sim}$ & 24 & 26,1 & 68 & 73,9 & \\
\hline Não & 50 & 56,2 & 39 & 43,8 & \\
\hline Doença de coluna & & & & & $<0,001^{*}$ \\
\hline Sim & 23 & 24,5 & 71 & 75,5 & \\
\hline Não & 51 & 58,6 & 36 & 41,4 & \\
\hline Diabetes & & & & & $0,001^{*}$ \\
\hline Sim & 5 & 15,2 & 28 & 84,8 & \\
\hline Não & 69 & 46,6 & 79 & 53,4 & \\
\hline
\end{tabular}

*Significância estatística (teste qui-quadrado). 


\section{DISCUSSÃO}

Esta pesquisa encontrou em seus resultados dados que são compatíveis com diversos outros estudos sobre a mesma temática. A estrutura interna da família tem se tornado cada vez mais diversificada, ao passo que várias gerações estão convivendo no mesmo ambiente familiar. ${ }^{12}$

A corresidência tem se mostrado a forma de viver em comunidade mais prevalente em levantamentos epidemiológicos, como o encontrado neste estudo e corroborado por Teixeira, ${ }^{13}$ que encontrou alta prevalência de corresidência no estado do Piauí, e por Almeida ${ }^{14}$ que obteve valores semelhantes em Porto Alegre-RS. Tais informações apontam que a corresidência não é um fenômeno típico de regiões com menor renda, pois tanto em estados do Nordeste, com baixos indicadores econômicos, quanto na Região Sul, uma das mais ricas do Brasil, observou-se a corresidência como estratégia de sobrevivência do idoso.

Foi observado também um fenômeno que tem sido característico em pesquisas sobre o envelhecimento populacional brasileiro a feminização da velhice. Diversos estudos apontam tal constatação, a exemplo do realizado em Fortaleza-CE, ${ }^{15}$ onde mais da metade dos idosos entrevistados era do sexo feminino, cenário também visto na cidade de DiamantinoMT. ${ }^{16}$ A feminização da velhice pode ser atribuída à maior procura por serviços de saúde por parte da mulher, assim como à preocupação com a própria saúde, dentre outros fatores. ${ }^{17}$

A faixa etária observada no estudo foi relativamente alta, considerando que apenas a menor parcela dos idosos entrevistados tinha menos de 70 anos de idade. Outros estudos também encontraram características semelhantes, como o realizado por Clares et al., ${ }^{15}$ no qual menos da metade dos idosos se encontrava na faixa etária entre 60 e 70 anos de idade. Entretanto, não se pode afirmar que este seja um fenômeno unânime, pois no estudo feito por
Fontes et al., ${ }^{16}$ a maior parte dos idosos encontrase na faixa etária de 60-69 anos de idade. Esta tendência da longevidade reflete uma realidade mundial, na qual convergem nesse processo o contínuo avanço das ciências biomédicas e a melhoria do acesso à saúde, principalmente em sistemas universais, como no Brasil. ${ }^{4}$

$\mathrm{Na}$ análise do estado conjugal dos idosos, não houve concordância entre todos os estudos na descrição dessa variável. $\mathrm{Na}$ maioria deles, os resultados mais prevalentes são "casado/em união estável" ou "viúvo", conforme encontrado em Fortaleza-CE. ${ }^{15}$ Nessa capital, os resultados apontaram a maior parte de idosos casados, seguidos de viúvos, tal como visto em São Carlos-SP. ${ }^{18}$ Entretanto, existiram casos em que a presença de viúvos foi mais prevalente do que a presença de idosos casados, como no estudo realizado em um município da Região CentroOeste do Brasil, e no estudo feito em Jequié-BA. ${ }^{19}$

Nesta investigação populacional, a quase totalidade relatou ter filhos, dado encontrado também em outros estudos, como no realizado numa USF de Porto Alegre-RS. ${ }^{14}$ Ter filhos pode ser considerada uma garantia de cuidado e atenção ao idoso, assim como uma forma de apoio e proteção social e financeira.

Outro fator que também pode ser considerado pelo idoso como uma forma de proteção social e financeira é o recebimento de algum benefício previdenciário, seja aposentadoria ou pensão. Essa sensação de proteção pode advir do fato que é uma renda fixa do idoso, com grande impacto em suas condições de saúde. Neste estudo, a maioria dos idosos recebia benefício, assim como o observado numa cidade do Rio Grande do Sul. ${ }^{14} \mathrm{O}$ recebimento do benefício representa muitas vezes a única fonte de renda da família e, segundo o estudo de Clares et al., ${ }^{15}$ a aposentadoria representa uma melhoria das condições de vida dos idosos.

Além do perfil sociodemográfico, o conhecimento do ambiente familiar que abriga 
o idoso permite visualizar as características da corresidência e a forma com que esses sujeitos avaliam o fato de morar com a família. Apesar de ser uma importante ferramenta para o entendimento das condições que podem proteger ou deteriorar o estado de saúde da população idosa, os aspectos ligados à corresidência ainda são pouco estudados.

O cenário desta pesquisa mostra que mais da metade dos lares desses idosos era composto por três ou mais moradores. Da mesma forma, o estudo epidemiológico de Bertuzzi et al. ${ }^{20}$ evidenciou que $50,1 \%$ dos idosos habitam em residência com esse quantitativo de moradores. Em muitos estudos populacionais, observa-se a existência de arranjos trigeracionais sobrepondo-se aos demais tipos de arranjos familiares, assim como verificado no estudo SABE, realizado em São Paulo. ${ }^{21}$

São diversos os fatores que podem interferir na percepção do idoso com relação à sua vida. Os arranjos familiares, o nível socioeconômico, as condições de saúde, processos de saúde-doença, entre outros, podem ser alguns deles. A população estudada mostrou-se, em geral, satisfeita com a vida que tem. Esta variável também pode sofrer influências positivas do alto grau de satisfação do idoso no que se refere ao arranjo familiar em que habitam e com o bom convívio com esses coabitantes, corroborados pelos relatos de que a corresidência seja uma forma de vivência vantajosa para ambos. Isto pode tornar a família do idoso uma importante aliada na construção de um envelhecimento saudável, já que segundo Camarano et al., ${ }^{4}$ a família é o suporte social mais próximo do idoso.

A avaliação do perfil sanitário de uma população deve extrapolar o simples fato de classificar os idosos em portadores ou não portadores de enfermidades crônicas. Este grupo de patologias vem acometendo um número cada vez maior de indivíduos com as mais diferenciadas características, portanto é preciso utilizar outros conceitos para que se tenha uma real compreensão do nível de saúde..$^{19}$ Nesse contexto, a avaliação funcional surge como ferramenta útil na identificação das condições de saúde do idoso, tendo em vista que esta variável pode representar a maneira como a doença crônica impacta na capacidade desse indivíduo para realizar as atividades básicas e instrumentais da vida diária.

O presente estudo encontrou uma prevalência elevada de idosos com autorrelato de doenças crônicas, o que pode ser explicado pela considerável quantidade de sujeitos com mais de 70 anos de idade. Associadas ao processo de envelhecimento, ocorrem alterações funcionais que são próprias deste grupo etário, ocasionando maior predisposição ao surgimento de condições crônicas. ${ }^{21}$ Esta maior predisposição pode ser outra justificativa para a alta prevalência dessas doenças encontrada neste estudo, que também pode ser observada em outros levantamentos epidemiológicos sobre o envelhecimento, como o realizado em Maringá-PR ${ }^{22}$ e Fortaleza-CE. ${ }^{15}$

Este trabalho avaliou a capacidade dos idosos quanto às atividades instrumentais da vida diária e observou que mais da metade deles realizava as atividades sem necessitar de qualquer auxílio; além disso, quase a totalidade deles tinha independência para realizar atividades básicas da vida diária. Em Juiz de Fora-MG, Silva et al. ${ }^{23}$ encontraram valores semelhantes de idosos independentes para as atividades instrumentais propostas por Lawton. A avaliação funcional de atividades instrumentais é uma forma de medir a capacidade de um indivíduo realizar atividades mais elaboradas, que envolvam estado intelectual e interação social.

Comparando com levantamentos internacionais, observou-se que em Lisboa, Portugal, ${ }^{24}$ a maioria dos idosos se mostrou autônoma em suas atividades instrumentais. A manutenção dessa capacidade funcional pode ter implicações diretas na qualidade de vida dos idosos, por estar relacionada com a capacidade de o idoso ocuparse com trabalho, fazendo com que ele se sinta mais útil dentro de sua casa e na comunidade em que vive. ${ }^{25}$ Uma maneira de manter a capacidade funcional de um indivíduo, seja ele idoso ou não, 
é evitar fatores que comprometam sua saúde e, consequentemente, sua habilidade de executar atividades, sejam elas básicas ou instrumentais. ${ }^{17}$

Segundo Veras, ${ }^{26}$ assim como ocorreu a transição demográfica, ocorreu também a transição epidemiológica, em que se observa que o atual padrão de doença é composto sobretudo das patologias classificadas como crônicas não transmissíveis. Entre essas doenças estão a hipertensão arterial sistêmica (HAS), as doenças cardíacas, diabetes mellitus (DM), doenças de coluna, reumatismos, entre outras. Neste trabalho, a doença mais prevalente foi a HAS, seguida de doença de coluna, reumatismo, doença cardíaca e diabetes. A HAS também foi a mais prevalente em estudo feito em São Paulo$\mathrm{SP}^{27}$ e na capital cearense. ${ }^{15}$

"Doença de coluna", assim como na presente pesquisa, foi a segunda morbidade mais prevalente em idosos de Ribeirão Preto-SP. ${ }^{28} \mathrm{Na}$ investigação feita por Leite et al., ${ }^{29}$ a presença de reumatismos foi de 4,3\%, contrapondo-se ao alto percentual encontrado nesta pesquisa. A Pesquisa Nacional por Amostra de Domicílios (PNAD 2003), segundo a pesquisa de Alves et al., ${ }^{30}$ mostrou que $17,4 \%$ da população idosa apresentavam doenças cardíacas, taxa semelhante à encontrada neste estudo. Quanto à baixa prevalência de $\mathrm{DM}$, os estudos feitos em São Paulo-SP, ${ }^{27}$ João Pessoa-PB ${ }^{29}$ e Diamantino$\mathrm{MT}^{16}$ corroboram este artigo, apresentando baixos índices de prevalência.

A análise da autopercepção de saúde dos idosos estudados evidenciou que a maioria relatou um status negativo de saúde. Para a compreensão mais detalhada desta variável, a análise estatística inferencial demonstrou que os aspectos que apresentaram associação estatisticamente significante foram aqueles relacionados à saúde, não havendo relação com aspectos sociodemográficos ou que remetiam à corresidência.
A associação entre o autorrelato de saúde e a avaliação funcional para a realização das atividades da vida diária, encontrada nesta pesquisa, também foi evidenciada em estudo conduzido na China, no qual se percebeu que baixas pontuações na avaliação funcional, que indicam algum grau de dependência, estiveram associadas com autorrelatos negativos de saúde. ${ }^{31}$

As doenças crônicas estão fortemente ligadas à autopercepção de saúde dos idosos estudados. Corroborando este dado, encontrase o estudo realizado em Porto Alegre-RS, no qual Hartmman ${ }^{8}$ observou essa relação com associação estatística. A autora verificou que a presença de doenças crônicas fez com que os idosos percebessem de forma pior a própria saúde. $\mathrm{Na}$ mesma pesquisa, $\mathrm{Hartmman}^{8}$ encontrou forte evidência estatística da relação entre autopercepção negativa de saúde e HAS, reumatismo e doença de coluna ( $\mathrm{p}$-valor $<0,001)$.

A mesma relação foi constatada por Carvalho et $a{ }^{32}$ no estudo feito com base nos dados obtidos por meio da Pesquisa por Amostra de Domicílios realizada na região metropolitana de Belo Horizonte-MG, que entrevistou 963 pessoas com idade igual ou superior a 60 anos, mostrando associação estatisticamente significante entre doença crônica e autopercepção de saúde.

Também confirmando os dados desta pesquisa estão os achados em uma cidade do Vale do Rio dos Sinos-RS, em que Barcelos \& Oliveira ${ }^{33}$ puderam observar a relação "autopercepção de saúde x doenças crônicas". Os autores verificaram que a categoria "sem doenças crônicas" estava representada por um percentual expressivo dos idosos da amostra e puderam relacionar tal dado à boa autopercepção de saúde dessa população. Tal fato pode estar ligado à inserção do idoso na comunidade, ao seu convívio no meio social e ao estilo de vida ativo dos idosos pesquisados. 
A autopercepção de saúde é um medidor de grande relevância na avaliação dos aspectos relacionados à saúde da população idosa, tendo em vista sua sensibilidade para avaliar indiretamente aspectos ligados ao bem-estar e à qualidade de vida desse grupo etário. ${ }^{31} \mathrm{E}$, portanto, uma fonte confiável para elaboração de ações integradas que atuem sobre os fatores determinantes para uma autopercepção negativa de saúde, corrigindo as deficiências e suprindo as necessidades que a população apresenta, tendo em vista a promoção de saúde e o bem-estar do idoso, melhorando, consequentemente, sua qualidade de vida. Mostrase assim a importância do melhor conhecimento sobre este marcador para a saúde da população.

Este estudo aponta ainda a importância de um bom suporte social na velhice e suas implicações nos autorrelatos de satisfação com a vida. Os idosos, apesar de uma percepção de saúde negativa, mostraram-se satisfeitos com a vida que levam em família e este fator parece ser dominante sobre as características de saúde na determinação de um elevado grau de satisfação com a vida que levam.

Associados aos dados da saúde autorreferida, o perfil sociodemográfico, a caracterização do ambiente familiar, a avaliação funcional e a prevalência de doenças crônicas compõem um conjunto de informações úteis para a compreensão ampla do estado de saúde da pessoa idosa que vive em estado de corresidência.

O estudo apresentou limitações quanto à população, considerando que os resultados foram obtidos com uma população de idosos assistida pela ESF, não podendo ser ampliada para idoso em condições socioeconômicas que não utilizam esses serviços de saúde. Além disso, aponta-se a possibilidade de ter ocorrido viés de memória na resposta ao questionamento quanto à variável "doenças autorreferidas", visto que o idoso pode ter relatado doenças que não apresentava, em vez de relatar doenças diagnosticadas.

\section{CONCLUSÃO}

A partir do estudo, pode-se concluir que esses idosos costumam viver em lares com três ou mais moradores, são do sexo feminino, têm mais de 70 anos de idade, são casados, com pelo menos um filho, recebem algum benefício previdenciário e se mostram satisfeitos com o arranjo familiar em que vivem, assim como com a vida que têm. Os idosos estudados julgam ser a corresidência uma forma de vivência vantajosa para eles e para quem mora com eles.

No que se refere às características de saúde, o estudo concluiu que os idosos apresentavam uma autopercepção negativa de saúde, mostraram-se independentes na realização de atividades básicas da vida diária e não necessitavam de auxílio na execução das atividades instrumentais da vida diária. Além disso, os sujeitos da pesquisa ainda referiram pelo menos uma doença crônica, sendo as mais frequentes a hipertensão e a doença de coluna.

Em relação a suas condições de saúde, esses idosos apresentaram uma autopercepção negativa da saúde, apesar de serem independentes para a realização de atividades básicas da vida diária (ABVD) e para atividades instrumentais da vida diária (AIVD). Essa autopercepção negativa teve associação estatisticamente significante com o autorrelato de alguma doença crônica.

A compreensão ampliada das condições de vida do idoso torna necessário compreender os fatores relacionados aos aspectos psicológicos, familiares e sociais, tendo em vista que o alcance de uma velhice bem-sucedida extrapola as condições funcionais e o perfil de morbidade dos idosos. Estar atento a essas nuanças do processo de envelhecimento, especialmente para os profissionais de saúde que lidam com o idoso no nível da atenção básica, remete aos preceitos da humanização do cuidado e da prestação de uma assistência integral. 


\section{REFERÊNCIAS}

1. Chaimowicz F, Greco DB. Dinâmica de institucionalização de idosos em Belo Horizonte, Brasil. Rev Saúde Pública 199; 33(5):454-60.

2. Alvarenga MRM, Oliveira MAC, Domingues MAR, Amendola F, Faccenda O. Rede de suporte social do idoso atendido por equipes de Saúde da Família. Ciênc Saúde Coletiva 2011;16(5):2603-11.

3. Carvalho IMM, Almeida PH. Família e Proteção Social. São Paulo Perspec 2003;17(2):109-22.

4. Camarano AA, Kanso S, Mello JL, Pasinato MT. Famílias: espaço de compartilhamento de recursos e vulnerabilidades. In: Camarano, AM, organizadora. Os Novos Idosos Brasileiros: muito Além dos 60?. Rio de Janeiro: IPEA; 2004. p. 137-67.

5. Camarano AA, El Ghaouri SK. Famílias com Idosos: ninhos vazios?. In: Associação Brasileira de Estudos Populacionais. $13^{\circ}$ Encontro da Associação Brasileira de Estudos Populacionais; 4 - 8 Nov 2002; Ouro Preto, MG: ABEP.

6. Freitas DHM, Campos FCA, Linhares LQ, Santos CR, Ferreira CB, Diniz BS, et al. Autopercepção de saúde e desempenho cognitivo em idosos residentes na comunidade. Rev Psiquiatr Clín 2010;37(1):32-5.

7. Jóia LC, Ruiz T, Donalísio MR. Grau de satisfação com a saúde entre idosos do município de Botucatu, Estado de São Paulo, Brasil. Epidemiol Serv Saúde 2008;17(3):187-94.

8. Hartmann ACVC. Fatores associados a autopercepção de saúde em idosos de Porto Alegre [tese]. Porto Alegre: Pontifícia Universidade Católica do Rio Grande do Sul, Instituto de Geriatria e Gerontologia, Programa de Pós-Graduação em Gerontologia Biomédica; 2008.

9. Pimenta FA, Amaral CS, Torres HG, Rezende Nilton. Autopercepção do estado de saúde em reformados e sua associação com o uso de serviços de saúde. Acta Med Port 2010;23(1):101-6.

10. Instituto Brasileiro de Geografia e Estatística, Diretoria de Pesquisa, Coordenação de Trabalho e Rendimento, Gerência de Pesquisa Anual. Pesquisa Nacional por Amostra de Domicílios PNAD de 2003: Questionário de pesquisa. [internet]. IBGE. [acesso em setembro de 2011]. Disponível em: http:// www.ibge.gov.br/home/estatistica/populacao/ trabalhoerendimento/pnad2003/questpnad2003.pdf

11. Universidade de São Paulo, Faculdade de Saúde Pública. Estudo SABE: Saúde, bem-estar e envelhecimento. Condições de vida e saúde dos idosos do município de São Paulo [Internet]. São Paulo [acesso em setembro de 2011]. Questionário. Disponível em: http://hygeia.fsp.usp.br/sabe/Artigos/ Questionario_2006.pdf

12. Mota FRN, Oliveia ET, Marques MB, Bessa MEP, Leite BMB, Silva MJ. Família e redes sociais de apoio para o atendimento das demandas de saúde do idoso. Esc Anna Nery Rev Enferm 2010;14(4):833-8.

13. Teixeira SM. Família e as formas de proteção social primária aos idosos. Rev Kairós 2008;11(2):59-80.

14. Almeida JP. Arranjos familiares de idosos residentes na área de atuação de uma estratégia de Saúde da Família de Porto Alegre-RS [trabalho de conclusão de curso]. Porto Alegre: Universidade Federal do Rio Grande do Sul; 2011.

15. Clares JWB, De Freitas MC, De Almeida PC, De Galiza FT, Queiroz TA. Perfil de idosos cadastrados numa Unidade Básica de Saúde da Família de Fortaleza-CE. Rev RENE 2011;12(n. esp.):988-94.

16. Fontes KCFQ, Pissolato STC, Costa IG. Doenças crônicas não transmissíveis em idosos de uma Unidade de Saúde da Família em Diamantino-MT. Rev Matogrossense Enferm 2010:1-15.

17. Veras R. Envelhecimento populacional contemporâneo: demandas, desafios e inovações. Rev Saúde Pública 2009;43(3):548-44.

18. Pavarini SCI, Luchesi BM, Fernandes HCL, Mendiondo MSZ, Filizola CLA, Barham EJ, et al. Genograma: avaliando a estrutura familiar de idosos de uma unidade de saúde da família. Rev Eletr Enferm [Internet] 2008 [acesso em 15 ago 2012];10(1):39-50. Disponível em: http://www.fen.ufg. br/revista/v10/n1/v10n1a04.htm

19. Torres GV, Reis LA, Reis LA, Fernandes MH, Xavier TT. Relação entre funcionalidade familiar e capacidade funcional de idosos dependentes no município de Jequié (BA). Rev Baiana de Saúde Pública 2010;34(1):19-30.

20. Bertuzzi, D, Paskulin LGM, Morais EP. Arranjos e rede de apoio familiar de idosos que vivem em uma área rural. Texto \& Contexto Enferm 2012;21(1):158-66.

21. Duarte YAO, Lebrão, ML, Lima FD. Contribuição dos arranjos domiciliares para o suprimento de demandas assistenciais dos idosos com comprometimento funcional em São Paulo, Brasil. Rev Panam Salud Publica 2005;17(5/6):370-8.

22. Zavatini MA, Obreli-Neto PR, Cuman RKN. Estratégia Saúde da Família no tratamento de doenças crônico-degenerativas: avanços e desafios. Rev Gaúcha Enferm 2010;31(4):647-54. 
23. Silva SLA, Vieira RA, Arantes P, Dias RC. Avaliação de fragilidade, funcionalidade e medo de cair em idosos atendidos em um serviço ambulatorial de Geriatria e Gerontologia. Fisioter Pesqui 2009;16(2):120-5.

24. Silva JSV. Funcionalidade e bem-estar psicológico em idosos residentes na comunidade: um estudo exploratório [dissertação]. Lisboa: Faculdade de Psicologia; 2010.

25. Rosa TEC, Benício MHD, Latorre RDO, Ramos LR. Fatores determinantes da capacidade funcional entre idosos. Rev Saúde Pública 2003;37(1):40-8.

26. Veras RP. Um modelo em que todos ganham: mudar e inovar, desafios para o enfrentamento das doenças crônicas entre os idosos. Acta Sci Hum Soc Sci 2012;34(1):3-8

27. Felipe LK, Zimmermann A. Doenças crônicas degenerativas em idosos: dados fisioterapêuticos. Rev Bras Promoç Saúde 2011;24(3):221-7.

28. Pedrazzi EC, Rodrigues RAP, Schiaveto FV. Morbidade referida e capacidade funcional de idosos. Cienc Cuid Saude 2007;6(4):407-13.
29. Leite CC, Rodrigues-Gonçalves MC, Rios-Asciutti LS, Leite-Cavalcanti A. Prevalência de doenças crônicas e estado nutricional em um grupo de idosos brasileiros. Rev salud pública 2009;11(6):865-77.

30. Alves LC, Leite IC, Machado CJ. Fatores associados à incapacidade funcional dos idosos no Brasil: análise multinível. Rev Saúde Pública 2010;44(3):468-78

31. Sereny MD, Gu D. Living arrangement concordance and its association with self-rated health among institutionalized and community-residing older adults in China. J Cross Cult Gerontol 2011;26(3):239-59.

32. Carvalho FF, Santos JN, Souza LM, Souza NRM. Análise da percepção do estado de saúde dos idosos da região metropolitana de Belo Horizonte. Rev Bras Geriatr Gerontol 2012;15(2):285-93.

33. Barcelos ARG, Oliveira LIE. Relações entre autopercepção de saúde, aspectos sociodemográficos e doenças crônicas não transmissíveis de idosas que frequentam um projeto social de uma cidade do Vale do Rio dos Sinos, RS. Rev Conhecimento 2011;3(2):1-17. 